\title{
Successful Treatment with Olanzapine of Psychosis in Dentatorubral-pallidoluysian Atrophy: A Case Report
}

\author{
Zui Narita ${ }^{1}$, Tomiki Sumiyoshi ${ }^{2}$ \\ ${ }^{1}$ Department of Psychiatry, National Center Hospital, National Center of Neurology and Psychiatry, ${ }^{2}$ Department of Clinical Epidemiology, \\ Translational Medical Center, National Center of Neurology and Psychiatry, Tokyo, Japan
}

\begin{abstract}
Patients with dentatorubral-pallidoluysian atrophy occasionally elicit psychosis. So far, one study reported first generation antipsychotics drugs may provide an effective treatment; however, there is no literature on the benefits of second generation antipsychotics. We report on a 44-year-old man with dentatorubral-pallidoluysian atrophy whose psychotic symptoms were effectively treated with olanzapine. Our observation suggests some second generation antipsychotics provide a therapeutic option for ameliorating psychosis in dentatorubral-pallidoluysian atrophy.
\end{abstract}

KEY WORDS: Progressive myoclonic epilepsies; Spinocerebellar degenerations; Psychosis; Antipsychotics.

\section{INTRODUCTION}

Dentatorubral-pallidoluysian atrophy (DRPLA), one of the spinocerebellar degeneration diseases, is a genetic autosomal dominant condition caused by an expansion of CAG repeats. ${ }^{1,2)}$ The morbidity of the illness in Japan is estimated approximately 0.2-0.7/100,000. ${ }^{3)}$ Although Burke et al. ${ }^{4)}$ reported that expanded CAG repeats occur more frequently in Japan compared to other countries, Warner et al. ${ }^{5)}$ argues that non-Japanese population may also be vulnerable to DRPLA, which would be more common than generally considered.

Patients with DRPLA typically show symptoms, such as epileptic seizures, myoclonus, ataxia, and dementia., ${ }^{6,7)}$ Also, these patients sometimes experience psychosis, but its treatment has yet to be established. For example, Adachi et al. ${ }^{8)}$ reported first generation antipsychotics drugs (FGAs), such as haloperidol and levomepromazine, ameliorated psychosis in DRPLA. However, there is no in-

Received: October 18, 2016/Revised: December 6, 2016 Accepted: December 8, 2016

Address for correspondence: Zui Narita, MD

Department of Psychiatry, National Center Hospital, National

Center of Neurology and Psychiatry, 4-1-1, Ogawahigashi,

Kodaira, Tokyo, Japan

Tel: +81-42-341-2711, Fax: +81-42-346-1944

E-mail: zuinarita@ncnp.go.jp

ORCID: https://orcid.org/0000-0001-7022-2141 formation on the efficacy of second generation antipsychotics (SGAs), based on the search of MEDLINE and PsycINFO.

Here, we report on a patient with DRPLA whose psychotic symptoms were effectively treated with olanzapine.

\section{CASE}

The patient was a 44-year-old Japanese man. One year before consulting our hospital, cerebellar ataxia and choreoathetosis had developed. Also, he had started hearing weird voices and showing hyperactivity. These symptoms had not been sufficiently improved with sodium valproate at 1,200 mg/day, which rendered him to consult us. Brain imaging with computed tomography demonstrated cerebellar and brainstem atrophy, and genetic examination confirmed the diagnosis of DRPLA. His mother also received the diagnosis of DRPLA, and has been hospitalized for many years because of severe physical conditions.

When the patient was admitted to our hospital, the hallucinatory-paranoid state dominated. For example, he believed that his "younger sister" married a 65-year-old man, although he actually does not have a sister. Also, he complained about auditory hallucinations, for example, hearing sounds as if a surgeon was performing an operation.

Psychotic disorder due to DRPLA was diagnosed in the

(C) This is an Open-Access article distributed under the terms of the Creative Commons Attribution Non-Commercial License (http://creativecommons.org/licenses/by-nc/4.0) which permits unrestricted non-commercial use, distribution, and reproduction in any medium, provided the original work is properly cited. 
patient, and we started treatment with olanzapine, at 2.5 $\mathrm{mg} /$ day (before sleep) as initial dose. We titrated it to 7.5 mg once a day to effectively ameliorate his symptoms. Four weeks after the start of medication, the hallucinatory-paranoid state was improved, so that he no longer heard weird sounds or elicited hyperactivity. Throughout the treatment, he did not experience adverse effects, such as extrapyramidal syndromes (EPS), and follow-up blood test results were normal.

When the patient consulted us for the first time, he did not understand the necessity of treatment for psychotic symptoms. At the time of discharge, he became more observant and insightful enough to be adherent to medications. He is seeing us regularly and keeping treatment with olanzapine at $7.5 \mathrm{mg} /$ day.

\section{DISCUSSION}

To our knowledge, this is the first report of the ability of olanzapine, one of the SGAs, to improve psychotic symptoms associated with DRPLA. When the patient showed the hallucinatory-paranoid state, he was fully conscious, not delirious, a condition similar to the patient with schizophrenia. In fact, schizophrenia has been sometimes misdiagnosed in patients with psychosis due to DRPLA. ${ }^{9}$ (10) To identify DRPLA, it is necessary to carefully examine the history of present illness. In our patient, neurological and psychotic symptoms started simultaneously, consistent with the diagnosis of psychotic disorder due to DRPLA. ${ }^{11)}$ He had been treated with sodium valproate in previous hospitals soon after complaining about these symptoms, indicating the absence of duration of untreated psychosis.

The relative paucity of information on psychosis in DRPLA may be due to the low incidence of the disease itself. On the other hand, Adachi et al. ${ }^{8)}$ reported approximately $10 \%$ of patients with DRPLA complain psychosis, suggesting the importance of information on its treatments. For patients with organic mental disorders, antipsychotics are not always recommended because adverse effects tend to occur frequently. ${ }^{12)}$ The use of FGAs is generally associated with side effects, such as EPS, which are less frequent with SGAs. ${ }^{13,14)}$ Therefore, we administered olanzapine to the patient, as it has favorable profile in terms of the incidence of EPS compared to other antipsychotics. ${ }^{15)}$ Hence, the effectiveness of olanzapine, not associated with adverse effects, observed here, may provide a promising strategy for treating psychosis in DRPLA.

A limitation of this study is that we did not use quantative measures, such as the Positive and Negative Syndrome Scale and the Brief Psychiatric Rating Scale, to evaluate severity of psychosis. This issue warrants further investigations for the benefit of SGAs to treat psychosis with DRPLA.

\section{REFERENCES}

1. Ikeuchi T, Koide R, Tanaka H, Onodera O, Igarashi S, Takahashi $\mathrm{H}$, et al. Dentatorubral-pallidoluysian atrophy: clinical features are closely related to unstable expansions of trinucleotide (CAG) repeat. Ann Neurol 1995;37:769-775.

2. Koide R, Ikeuchi T, Onodera O, Tanaka H, Igarashi S, Endo K, et al. Unstable expansion of CAG repeat in hereditary dentatorubral-pallidoluysian atrophy (DRPLA). Nat Genet 1994; 6:9-13.

3. Inatsuki G, Kumagai K, Naito H. /Geographical distribution of origins of DRPLA family in Japan, and prevalence of DRPLA in Niigata prefecture]. Seishin Igaku 1990;32:1135-1138. Japanese.

4. Burke JR, Ikeuchi T, Koide R, Tsuji S, Yamada M, Pericak-Vance MA, et al. Dentatorubral-pallidoluysian atrophy and Haw Rever syndrome. Lancet 1994;344:1711-1712.

5. Warner TT, Williams LD, Walker RW, Flinter F, Robb SA, Bundey SE, et al. A clinical and molecular genetic study of dentatorubropallidoluysian atrophy in four European families. Ann Neurol 1995; 37:452-459.

6. Naito H, Oyanagi S. Familial myoclonus epilepsy and choreoathetosis: hereditary dentatorubral-pallidoluysian atrophy. Neurology 1982;32:789-807.

7. Takahashi H, Ohama E, Naito H, Takeda S, Nakashima S, Makifuchi T, et al. Hereditary dentatorubral-pallidoluysian atrophy: clinical and pathological variants in a family. Neurology 1988;38:1065-1070.

8. Adachi N, Arima K, Asada T, Kato M, Minami N, Goto Yi, et al. Dentatorubral-pallidoluysian atrophy (DRPLA) presenting with psychosis. I Neuropsychiatry Clin Neurosci 2001; 13:258-260.

9. Naito $\mathrm{H}$, Ohama E, Nagai $\mathrm{H}$, Wakabayashi M, Morita M, lkuta F. [A family of dentatorubropallidoluysian atrophy (DRPLA) including two cases with schizophrenic symptoms]. Psychiatr Neurol Jpn 1987;74:871-897. Japanese.

10. Adachi N, Onuma T, Akashi T, Arima K, Asada T, Kato M, et al. [Four cases of progressive myoclonus epilepsy with paranoid state]. Seishin Igaku 1992;34:745-750. Japanese.

11. American Psychiatric Association. Diagnostic and Statistical Manual of Mental Disorders, fifth ed. Arlington, VA: American Psychiatric Association;2013. p.114-115.

12. Shoji Y, Uchimura N. /Clinical application of second genera- 
tion antipsychotics to organic, including symptomatic, mental disorders-possibility of second generation antipsychotics to adaptiatino expansion for delirium and/or higher brain disorder]. Rinsho Seishin Yakuri 2009;12:679-688. Japanese.

13. Halstead SM, Barnes TR, Speller JC. Akathisia: prevalence and associated dysphoria in an in-patient population with chronic schizophrenia. Br J Psychiatry 1994;164:177-183.

14. Glazer WM. Expected incidence of tardive dyskinesia asso- ciated with atypical antipsychotics. J Clin Psychiatry 2000;61: Supp/ 4:21-26.

15. Carlson CD, Cavazzoni PA, Berg PH, Wei H, Beasley CM, Kane JM. An integrated analysis of acute treatment-emergent extrapyramidal syndrome in patients with schizophrenia during olanzapine clinical trials: comparisons with placebo, haloperidol, risperidone, or clozapine. J Clin Psychiatry 2003;64: 898-906. 